\title{
A Novel Clustering Algorithm Based on Agent Technology for VANET
}

\author{
Samira Harrabi \\ ENSI University of Manouba/SOIE, Campus universitaire de Manouba 2010, TUNISIA \\ E-mail: samira.harrabi@gmail.com \\ Ines Ben Jaafar \\ ESCT University of Manouba/SOIE, Campus universitaire de Manouba 2010, TUNISIA \\ E-mail: ines.benjaafar@gmail.com \\ Khaled Ghedira
}

ISG University of Tunis/SOIE, 41 Avenue de la liberté Bouchoucha, TUNISIA

E-mail: khaled.ghedira@isg.rnu.tn

Received: October 16, 2015 Accepted: December 15, 2015 Published: June 30, 2016

DOI: 10.5296/npa.v8i2.8434

URL: http://dx.doi.org/10.5296/npa.v8i2.8434

\begin{abstract}
Vehicular Ad-hoc Network (VANET) is a sub-family of Mobile Ad-hoc Network (MANET).The means goal of VANET is to provide communications between nearby nodes or between nodes and fixed infrastructure. Despite that VANET is considered as a subclass of MANET, it has for particularity the high mobility of vehicles producing the frequent changes of network topology that involve changing of road, varying node density and locations of vehicles existing in this road. That's why, the most proposed clustering algorithms for MANET are unsuitable for VANET. Various searches have been recently published deal with clustering for VANETs. But most of them are focused on minimizing network overhead value, number of created clusters and had not considered the vehicles interests which defined as any related data used to differentiate vehicle from another (such as traffic congestion, looking for free parking space, etc.). In this paper, we propose a novel clustering algorithm based on agent technology to solve the problems mentioned above and improve routing in VANET. Experimental part show promising results regarding the adoption of the proposed approach.
\end{abstract}




\section{I Macrothink}

Keywords: VANET, VANET, routing protocols, clustering, agent technology

\section{Introduction}

VANET is a particular class of MANET that provides communications between nearby vehicles, and between vehicles and fixed bases stations situated in communication environment as illustrated in "Fig. 1".

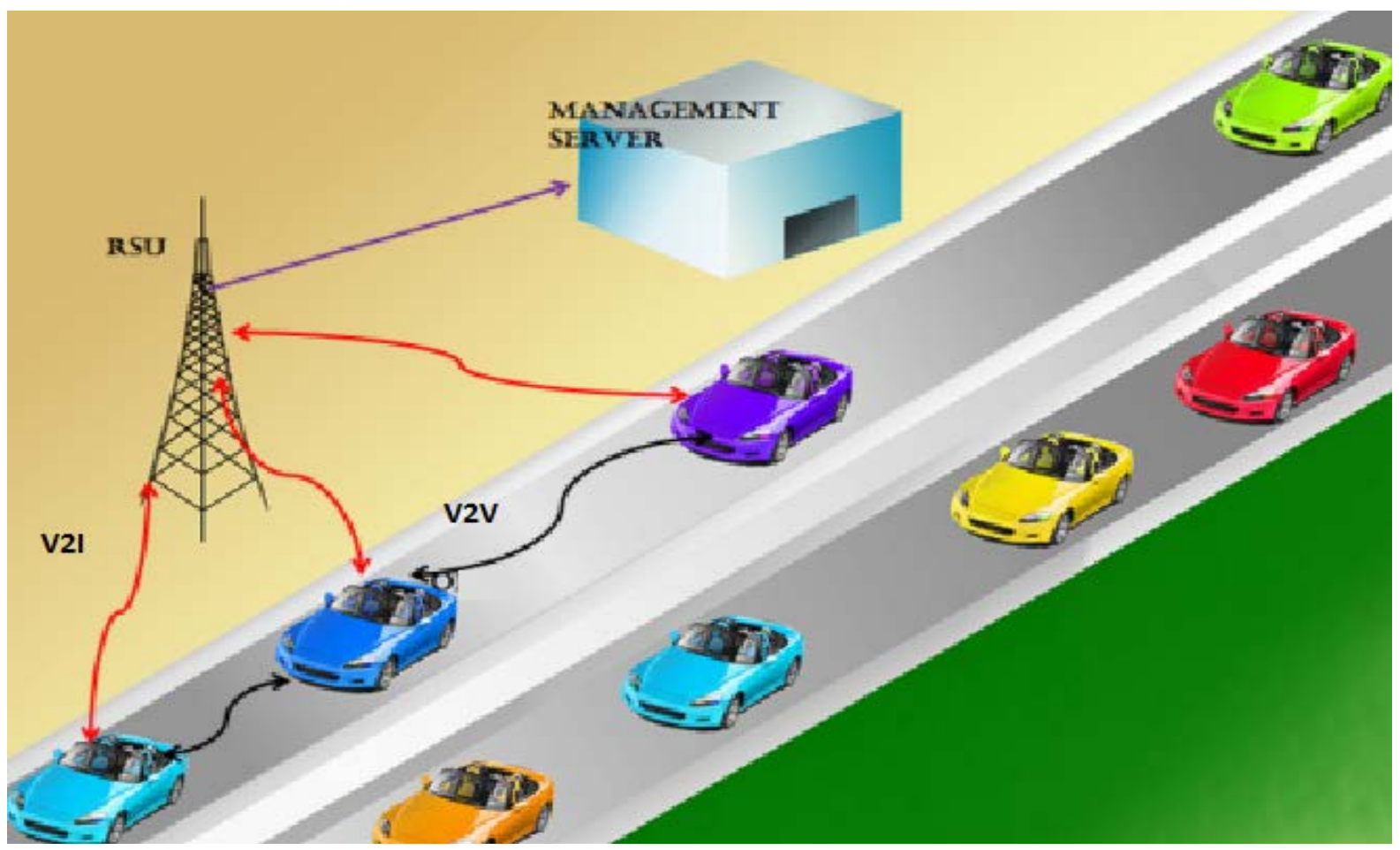

Figure 1. VANET's environment

The main purpose of VANET is to supply, firstly, the prevention and the road safety. The VANET can improve the road accident prevention, by alerting the driver of a dangerous situation. Secondly, VANET provide the traffic optimization, the car traffic can be largely improved due to the sharing of the data collected by vehicles. A car can, for example, be warned of a traffic jam.

So, we can classify these applications into two main categories. Safety applications like collision or obstacle alert, road conditions warning, merge assistance, deceleration warning, etc. where the main emphasis is on timely dissemination of safety critical alerts to nearby vehicles and comfort applications such as accessing emails, web browsing, audio and video streaming where the emphasis is on the availability of high bandwidth stable internet connectivity.

With the wireless technology becoming pervasive and cheap, the majority of vehicles have information concerning the outside environment constraints such as the map of the regions to be visited and the density of traffic on the road, etc. All this precious information can be exploited by the vehicles to get an adaptive decision about the choice of a suitable path. 
Furthermore, the moving vehicles can share information with the other vehicles to increase routing security. However, due to the mobility of the vehicles, it is hard to find the precise target for sending the information. This is due to the fact that with an increase in the density of the vehicles, collected information may not be accurate as the vehicles may change their position from a particular area. Consequently, a robust routing protocol is needed and required.

It has been demonstrated that clustering improves the performance of the VANETs and can be utilized in numerous applications as intelligent transportation systems (ITS) [1]. A lot of clustering algorithms for VANET have been developed. While most of these algorithms use the MANET clustering algorithms to form and maintain the clusters.

Therefore, these algorithms do not produce a stable clustering structure and the chosen path may not be the best. In cluster based routing protocols, vehicles form a cluster for managing a particular region. Because the vehicles may disappear or change the cluster to which they belong, their position also changes and links between vehicles can quickly disappear.

Consequently, to ensure uninterrupted route, the vehicles must be intelligent to be suitable for network environment and take autonomously the best decision when unexpected problem occurs such as topology changing, accident, traffic jam, varying in density of nodes based on the available information.

In this paper, we propose a new approach for cluster based routing algorithm. Each vehicle in the VANET is characterized by a defined interest as looking for parking space, accessing to Internet, accident alert etc. This paper presents two contributions: The first one is when an accident occurs on the road, the information about this accident should not be sent to each vehicle in the network but only to those nodes which are interested in receiving such data. Consequently the vehicle that detected the accident must send an alert message only for the nodes which are interested with this information.

So, we have to cluster the nodes by taking the interest or context information of the vehicles into account in order to optimize the information flow between nodes and decrease overall network traffic deployment.

The second contribution of this work is that the vehicle should be able to perceive the road and act autonomously in response to the accident alert message from the surrounding environment and take the best decision. It must be also intelligent to avoid accident problem and react quickly to events that may occur on environment communication. Since VANET has become an interesting research topic, several technologies have been proposed to overcome routing problem such as artificial intelligence [2], neural networks [3].

However, given that agent technology takes into account the aspects of cooperation, autonomy, distribution and intelligence [4], it has demonstrated a good behavior in a distributed and highly dynamic environment [5]. Consequently, we believe that assigning agent properties to vehicles may be appropriate to solve the afore-mentioned problems and improve routing performance in VANET. 
Agent technology is a field of computer science that is concerned with developing environment aware software agents that use intelligent algorithms allowing them to sense and respond to their environment, and taking, autonomously, an adaptive decision [4].

The rest of this paper is structured as follows. Section 2 presents the difference between MANET and VANET to highlight the using of MANET's routing protocol for VANET. Section 3 reviews related work. In section 4, we introduce the proposed algorithm. The simulation results are discussed in section 5. Finally, section 6 concludes the paper with a summary of the presented approach and presents our future work.

\section{The differences between MANET and VANET networks}

Despite the fact that VANET is a specific application of MANET, several studies have demonstrated that routing protocols designed for MANET are inadequate and have poor performance if they are used without any modification or improvement [6]. MANET is a set of nodes interconnected based on radio communication. These networks are a fully distributed nature and totally dynamic, in which each node must be able to configure itself without the need for any centralized management or no infrastructure previously deployed [7].

MANETs has no fixed infrastructure and they rely instead on ordinary nodes to perform message routing and network management functions. However, several characters such as driver behavior, the absence of constraint of energy, the availability of reliable location information, frequent changes in network topology due to the high mobility of vehicles make VANET behave differently from MANET. These unique network features can provide broken communication links between vehicles. Due to the design goals of MANET's routing protocols are not valid for high mobility networks, they cannot be directly applied in VANET [7].

The table 1 summarizes the differences between MANET and VANET [8].

Table1. MANET vs. VANET

\begin{tabular}{|l|l|l|}
\hline Network features & VANET & MANET \\
\hline Network topology & always changing & May change \\
\hline Mobility of nodes & Very high( up to 200km/h) & walking speed( up to $8 \mathrm{~km} / \mathrm{h}$ ) \\
\hline Density of nodes & Very high (limitless ) & Utmost 1000 nodes \\
\hline Constraint of energy & Absent & Limited \\
\hline Nodes movement & Irregular & regular \\
\hline Trajectory of node & road network (Predictable) & Randomly \\
\hline Stability route & Route can quickly disappear & more stable route \\
\hline
\end{tabular}

So, based on the above characteristics, we can justify the requirement to design a routing algorithm distinct from the traditional routing protocols. 


\section{Related work}

In recent years, several works reported in the literature deal with routing in VANETs among which the cluster- based routing protocols that show good prospects.

The authors in [9] proposed a multi-hop based cluster technique in order to progress stability. This method allows vehicles to choose their routes from one-hop neighbors. However, due to the mobility of nodes, it is hard to find the precise target for sending information.

In [10] the authors proposed a novel mobility-based clustering technique considering node mobility as metric for cluster formation process. Nevertheless, according to this method, all nodes make their cluster head decisions every clustering time seconds. Due to the instability of communications links, those decisions may not be the best.

In [11], the authors presented a stable cluster method in VANET based on speed and connectivity degree among nodes. Even that, the simulation results showed that the proposed scheme performs better in terms of cluster formation time and cluster life time, in our opinion, the maintenance process can take a lot of time because the vehicles can quickly disappear or change their cluster.

In [12], the authors developed another clustering method with an idea of taking only the public car as metric to form a group of vehicles. Nonetheless, this method is unsuitable in case where there is no public car.

VMaSC [13] is a novel clustering solution where the node that has the least mobility value will be selected as a cluster head. This approach based only on mobility value that requires a similar mobility patterns.

In [14], the authors studied the inter cluster communication and it used a double cluster head idea. The given method attempted to minimize energy consumption while energy constraint is a critical challenge for MANET and not for VANET.

The proposed approaches [15 and 16] are based on GPS system to form stable groups. They take into consideration location and mobility of nodes as two metrics that cannot be the ideal features due to the dynamic network topology.

In [17], the author presented a cluster-based routing algorithm to solve the broadcasting storm. In this method the cluster head is the faster vehicle. When an accident occurs, vehicle broadcasts an alert message includes the identifier and the location of the vehicle involving the accident. In this way, any vehicle can receive the notification even if it is not concerned by this alert which can increase network traffic.

In [18], the authors proposed a routing protocol for VANET to construct the route based on the traffic load, the density of the vehicle and the distance to the destination. The proposed method is based on junction geographic routing technique that sends massages along the routes. However, the main routing decision is made before junctions. 
In order to solve the problem of the mobility, a new mobility routing protocol for VANETs on the basis of a fixed direction at a high mobility value is published in [19]. The vehicle adopts this presented approach can obtains an IP address from the VANET via V2V architecture. But, due to the high mobility of nodes, we think that, an address conflict problem may be happen.

In [20], the authors proposed a new cluster-chain construction method. In this scheme, the authors considered, only, the distance from a cluster head to its neighbor as a factor to select the cluster head.

A localization method is presented in [21] where each vehicle evaluates its position based on a message that broadcasted by pairs of road side units (RSUs). This study attempted to develop routing performance based on localization of the vehicles that are currently on the road and their direction as well which are not guaranteed metrics in a highly dynamic environment.

An efficient clustering algorithm for clustering in VANETs is proposed in [22]. The authors considered different factors such as entropy, the direction of vehicle and the number of neighbors to perform the clustering of vehicles in an exact area. But like various clustering approach, this given solution used the GPS system.

The work proposed in [23] describes an adaptive connectivity aware routing in VANETs. The authors demonstrated the choice of the optimal path based on collecting data from diverse regions. Due to the fact that the density of nodes is varying, the collected information may not be precise.

In order to control and manage each network topology, the authors proposed in [24] an algorithm to structure the topology of diverse wireless sensor networks to coexist in the same environment. The given algorithm demonstrated good performance in terms of bandwidth consumption.

However, in these studies one of the vehicles which are cluster head takes all the decisions on behalf of the other vehicles. Consequently, as the topology changes, these decisions may become not valid.

The most of aforementioned clustering approaches seek to minimize network overhead as well as number of cluster heads, and so on. We consider that all this is of secondary importance and that the principal aim must be the uninterrupted links between vehicles and avoiding the dropped packets as possible even at the highest overhead value.

Due to the mobility of nodes in VANETs, the backbone must be continuously reconstructed in a timely fashion, so the research on more distributed, adaptive and intelligent protocol becomes more than critical. For that, agent technology has become an exciting and promising research area for vehicular ad-hoc.

Previously in [25], we have improved a proactive routing protocol to make it suitable for vehicular environment based on a multi-agent system. We have tested our approach that is called MA-DSDV by comparing its performances with DSDV routing protocol. Nevertheless, 
on one hand, MA-DSDV didn't take into consideration the vehicular interest. On other hand, and as it is a proactive routing protocol, in MA-DSDV, the routing overhead is elevated. One method to make routing more efficient is clustering technique.

For this raison, we have proposed in [26] an idea of cluster-based architecture by integrating the agents and taken the context information into account.

However, in this paper, we improve the given architecture by changing the number of main cluster members as well as the selection metrics of each one. In addition, in this paper simulation results are presented to study the performance of our proposed approach.

\section{Proposed clustering algorithm}

In this section, a Novel Clustering Algorithm Based on Agent Technology denoted (NCABAT) is presented to deal with routing in VANETs. Our approach is motivated, firstly, by the fact to assign agent properties to vehicles.

Secondly, we have to cluster the nodes by taking the context information of the vehicles into account in order to optimize the information flow between nodes and decrease overall network traffic deployment.

In [27], the authors defined the context as any related data that can be used to differentiate the situation of vehicles (such as vehicle identifier (ID), geographical location. left Lane, Right Lane etc.).

\subsection{Agent technology}

The problem we address here is the intrinsic properties of VANETs that make MANET's routing protocols unsuitable for VANET. In fact, routing protocol for this type of networks must be robust to ensure route stability and uninterrupted communication. The needed protocol must also be intelligent and to avoid dropping packets, the required method must react quickly to events that may occur such as topology changing or varying in density of nodes.

The idea of using agent technology for routing in vehicular networks has recently been explored in some works [28, 29 and 30].

Agent technology is a new paradigm from the field of artificial intelligence. An agent is an autonomous entity, real or abstract, which is able to perceive the environment in which they operate and dynamically react to the incumbent states that may occur.

An agent is capable to communicate with others agents in order to achieve its goals for which it was designed [4]. An agent has two special properties: orthogonal and mandatory characters which distinguish them from the traditional programs. The orthogonal properties are mobile, believable, communicative and learner. The mandatory properties are autonomous, reactive, proactive and temporally continuous. Consequently, a routing protocol based on this program seems appropriate to solve the afore-mentioned problems. 


\subsection{NCABAT overview}

As shown in Fig. 2", the main goal of the given approach is to divide the vehicles of VANETs into different disjoint groups where each vehicle is replaced by an agent. In our approach, we mainly focus on how to form a cluster where all agents have the same contextual information rather than how to select the main elements of each cluster. In literature, the term "contextual information” or "context” have different interpretations.

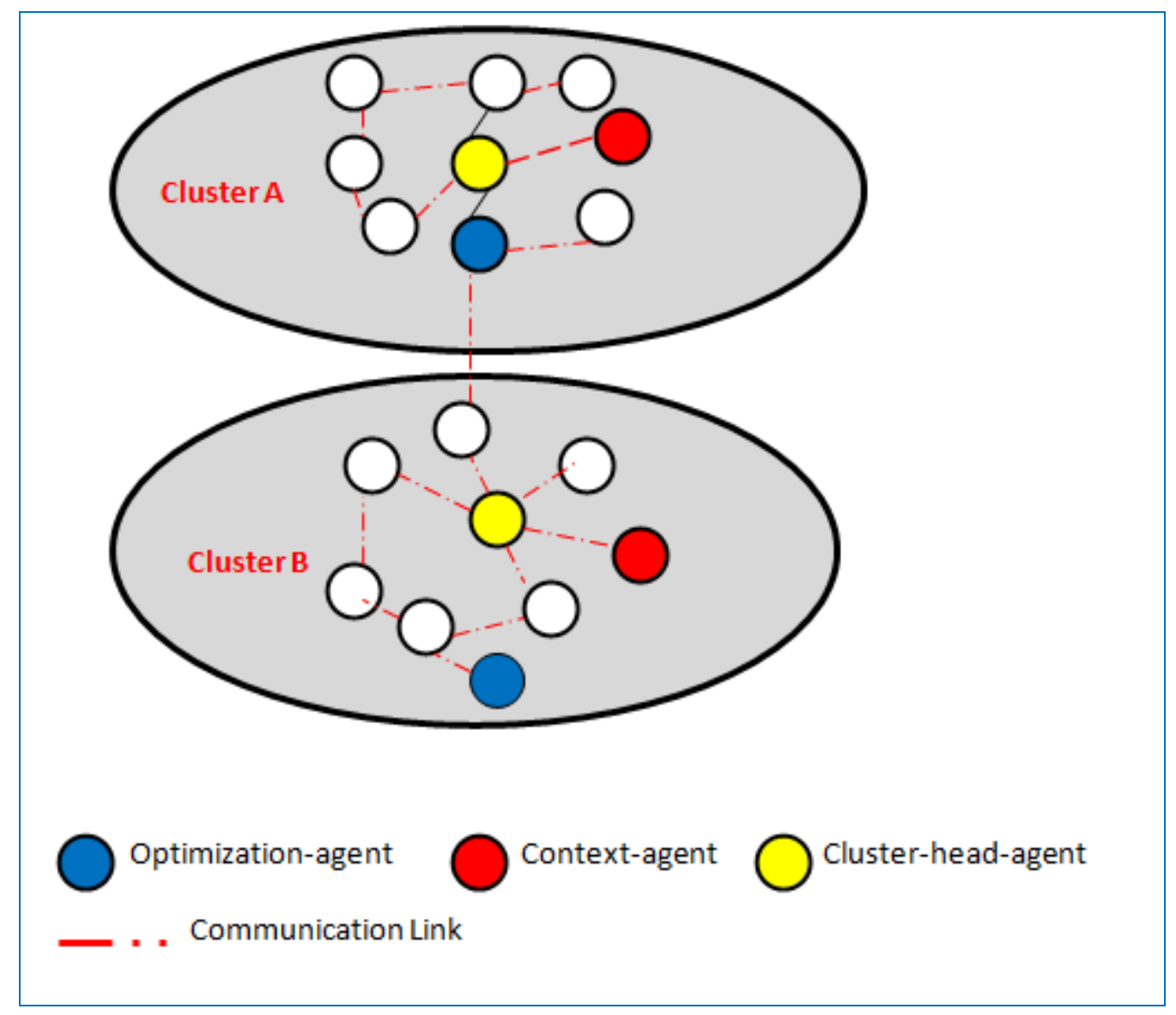

Figure 2. Cluster structure in NCABAT

In our solution, we consider that the vehicular interest is the character that can differentiate a vehicle from another. Indeed, in a communication environment, each vehicle has its purpose; it resorts to internet, either to buy a ticket for parking space, play games online, move to right or move to left etc. So, all these routing wishes define the vehicular interests. Each cluster has three vital agents which are:

\subsubsection{Cluster-head-agent}

It is the manager of its cluster. It has the responsibility of providing connection for its neighboring agents. 


\subsubsection{Context-agent}

It is responsible for collecting the context information. When an agent comes within a transmission- range distance from a nearby cluster-head, the context-agent checks if it has the same interest.

\subsubsection{Optimization-agent}

To minimize messages and traffic congestion, the messages must be communicated to the concerned cluster which is formed on a contextual information basis. Optimization-agent is the vehicle that can listen to the different vehicles of two adjacent clusters and provide communication between them.

\subsection{Cluster formation procedure}

In our approach, each agent enters the network in a "non-clustered" state. To declare its existence to its neighbors, every agent transmits its routing data which represents a unique and different identifier (ID), routing interest and current state.

Upon receiving this data, each agent compares its own interest with its neighbor's. If it is the same context and if an agent distinguishes that its own (ID) is the lowest of all its neighbors, as it can be seen in line 9 of pseudo-algorithm 1, the agent changes its state to “cluster-head-agent”.

Pseudo- Algorithm 1: Cluster-head -selection

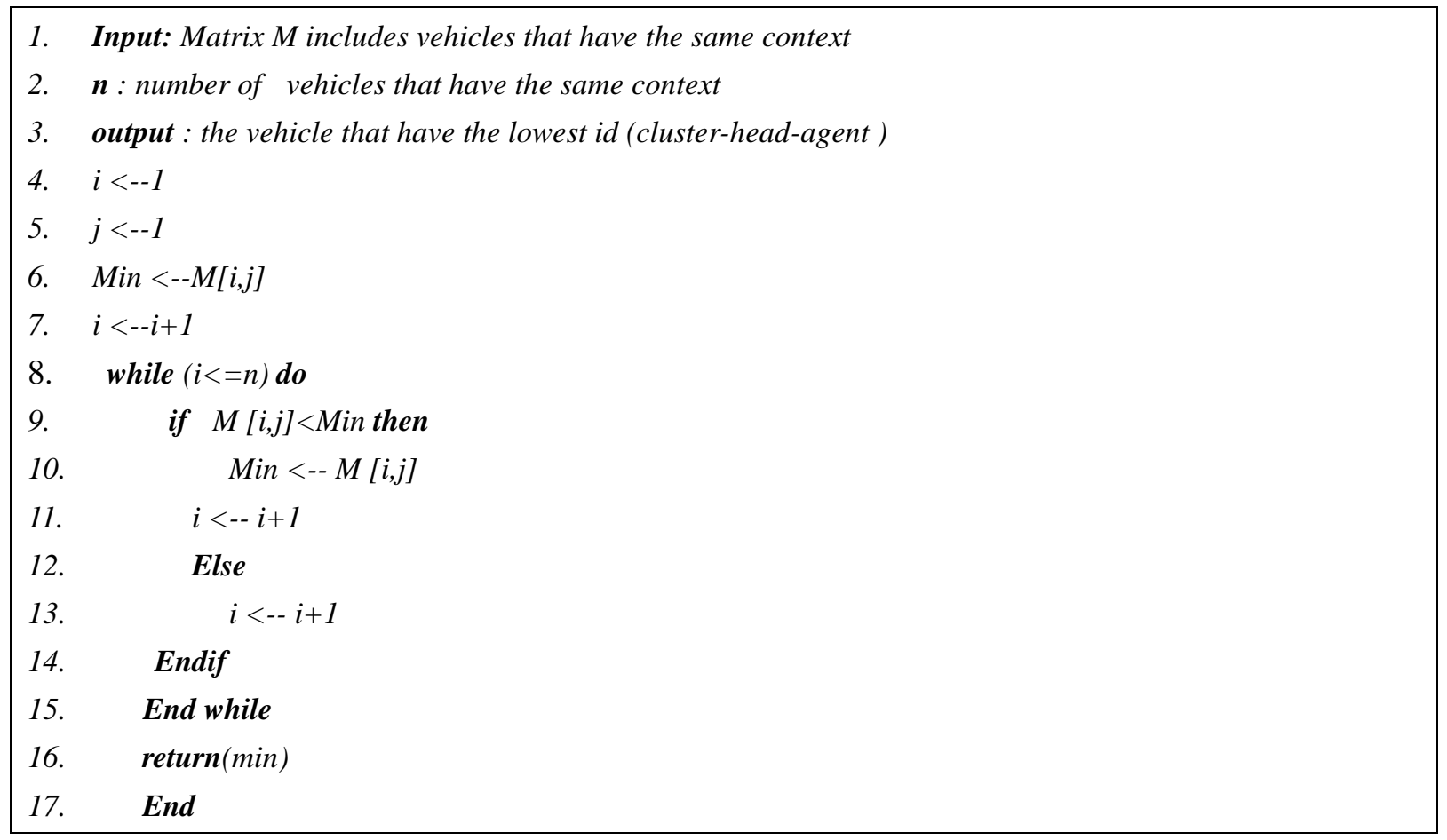

But if its own (ID) is the highest as shown in line 8 of the below code, then it declares itself as "context-agent". 
Pseudo- Algorithm2: Context-agent selection

1. Input: Matrix M includes vehicles that have the same context

2. $\boldsymbol{n}$ : number of vehicles that have the same context

3. output : context-agent

4. $i<--1 \quad j<--1$

5. $M a x<--M[i, j]$

6. $i<--i+1$

7. While $(i<=n)$ do

8. if $M[i, j]>\operatorname{Max}$ then

9. $\operatorname{Max}<-M[i, j]$

10. $i<--i+1$

11. Else

12. $i<--i+1$

13. End if

14. End while

15. Return (Max)

16. End

To ensure interconnections among neighbor clusters, the optimization-agent is the vehicle that is located in the nearest position to the neighboring cluster-head-agent. The simplest method to determine the nearest neighbor is the coordinates (X, Y) determined by the GPS system.

Indeed, we assume that the vehicle which has the highest value of $\mathrm{Y}$ will be elected according to the peudo-algorithm3, by the cluster-head agent, as an optimization-agent. The other agents in the cluster which have one-hop link to the cluster-head-agent are called the Ordinary Cluster Members (OCM).

\section{Pseudo- Algorithm 3: Optimization-agent selection}

1. Input: Matrix M includes vehicles that have the same context

2. $n$ : number of vehicles that have the same context

3. output : node that have the highest $Y$ (optimization-agent)

4. $i<--1$

5. $j<--5 \quad / *$ the position of $Y$ is the fifth box in each matrix line*/

6. $M a x<-M[i, j]$

7. $i<--i+1$

8. while $(i<=n)$ do

9. If $M[i, j]>$ Max then

10. $\quad \operatorname{Max}<--M[i, j]$

11. $i<--i+1$

12. Else

13. $i<--i+1$ 


\section{I Macrothink}

\section{Endif}

\section{End While}

16. $\operatorname{return}($ Max)

17. End

To inform its neighbors, each elected agent must broadcast a message containing its new state, its ID as well as the identifier of its clusters (ID_Cluster) (as shown in figure 2). In spite of the fact that the topology of VANET is dynamic, the network topology can be changed over time. As a result, cluster maintenance is needed. In our approach, the cluster formation procedure will be triggered when a vehicle leaves a cluster and moves to another neighboring cluster or when a "non-clustered" agent enters the network looking to join a cluster.

\subsection{Routing scenario}

In this section, we demonstrate the importance of taking into account the context information. Otherwise, the message would not be delivered to all neighboring agents (Broadcast), but only to a set of vehicles (Multicast).

The idea behind this choice is to improve the routing performance based on a multicast technique rather than a broadcast mode. As shown in "Fig. 3", each agent in the cluster 1 is interested in the Left line.

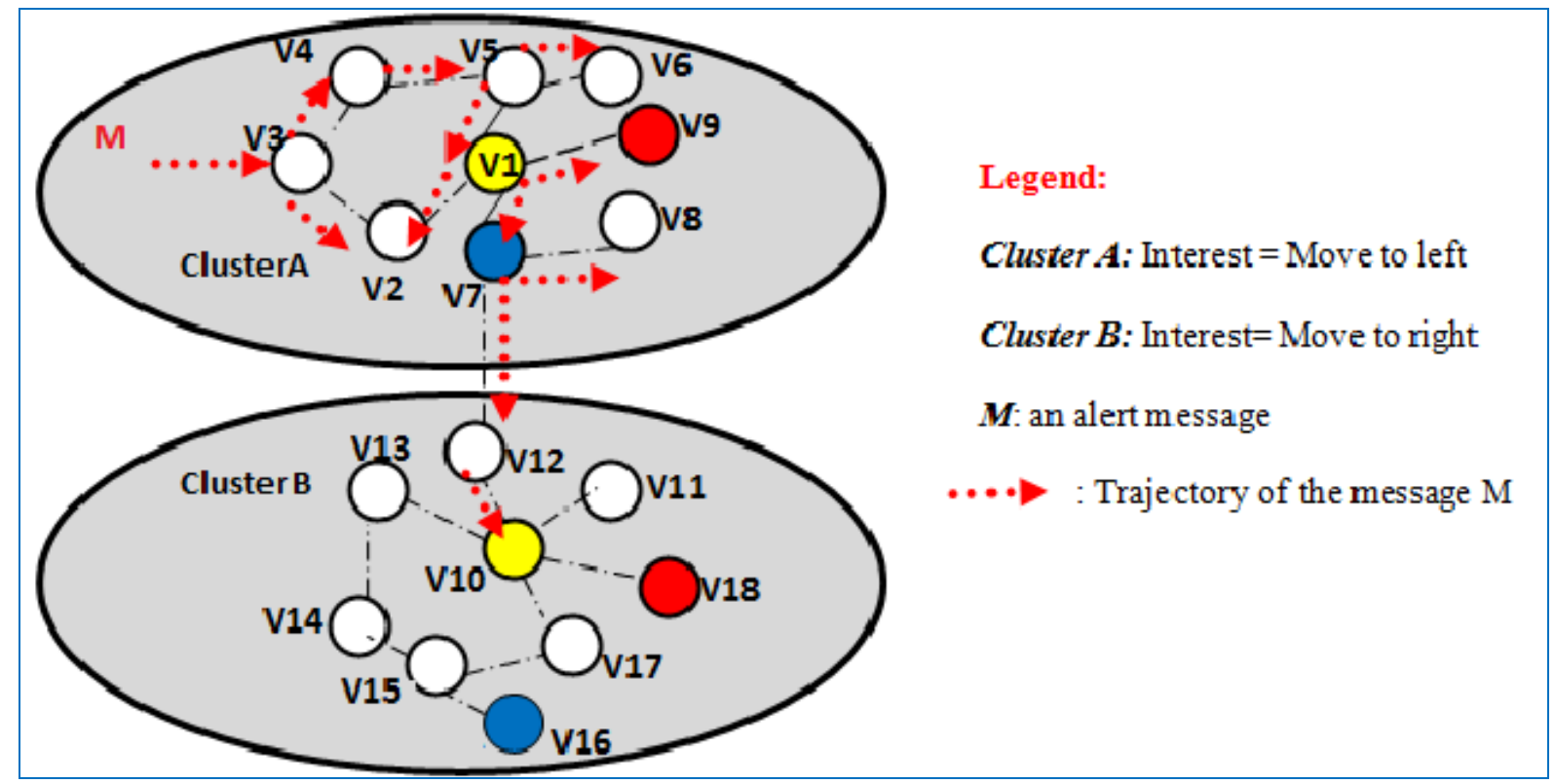

Figure 3. Broadcast routing

We assume that V3 was already informed by a vehicle on the left lane about an accident problem. So, if we don't take the context into consideration, V3 broadcasts the message to the next hop neighbors. In addition, agents blindly rebroadcast every message they receive without applying additional control mechanisms. This method is called "Flooding" [31]. This process continues until the request packet reaches all neighboring agents. 
As a result, even- though the agents of cluster 2 are not concerned by the accident alert, they have received the message which can increase the network traffic and decrease the routing performance.

According to our approach, the alert message routes to the interested agents, V3 forwards the packets to the next hop along the way to the cluster-head-agent. The cluster-head-agent forwards the packet to its optimization agent which in turn, will forward it to the concerned neighboring. This process continues until the request packet reaches either an intermediate agent or the destination agent itself as illustrated in "Fig. 4".

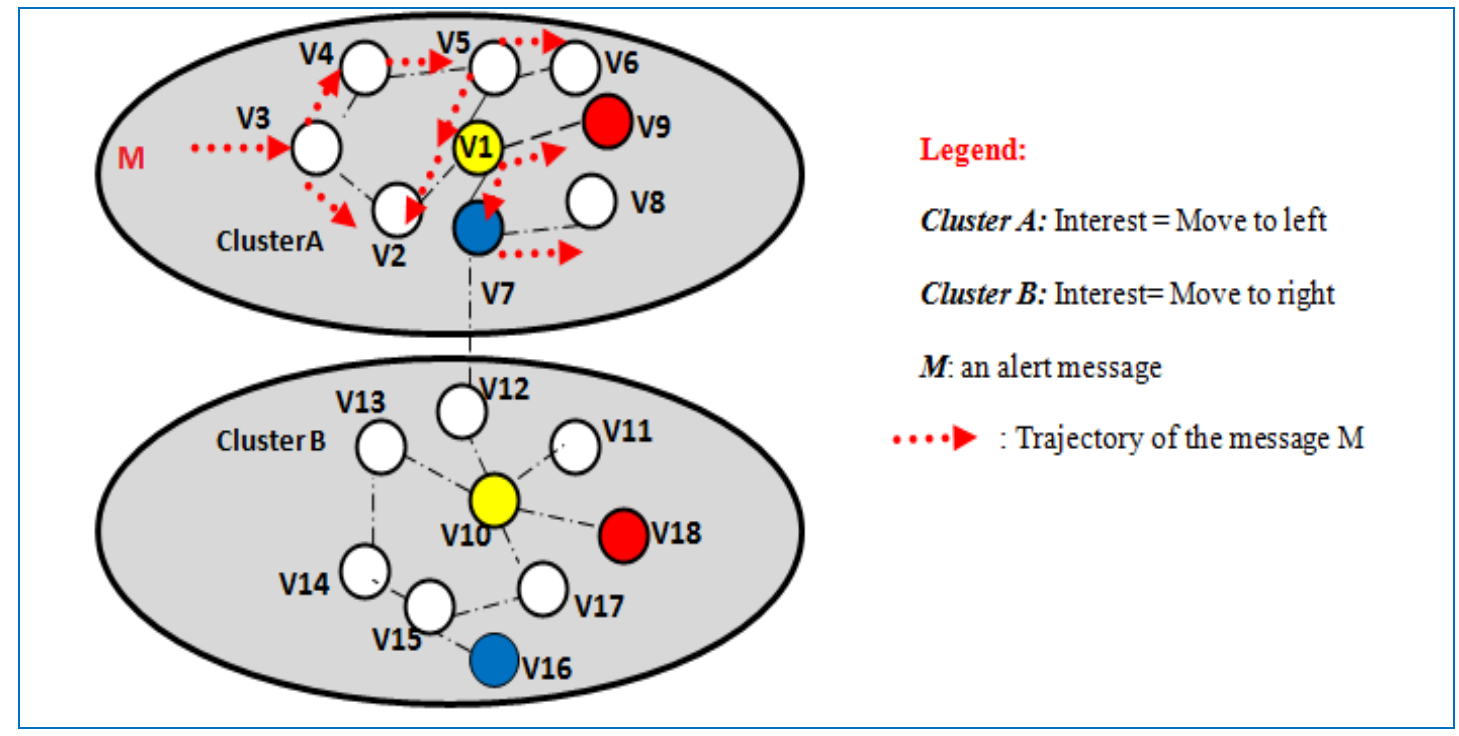

Figure 4. Multicast routing

In the following code presented in the pseudo-algorithm 4, we demonstrate the process of sending an alert message illustrated in the "Fig. 4".

Pseudo- Algorithm 4: sending message (Message = =”Alert message”)

1. Interest : define the interest of vehicle

2. State: the current state of vehicle $\mathrm{Vi}$

3. $\quad \mathrm{N}$ : the neighbors of $\mathrm{Vi}$

4. For each i in $N$ do

5. Interest $[\mathrm{i}]==$ Message

6. Send( Message, $\mathrm{Vi}, \mathrm{i})$

7. Else

8. Drop(Message)

9. End if

10. End for 


\section{Simulation results and performance comparison}

This section presents the performance evaluation of our approach. The proposed approach has been simulated using JADE Framework (Java Agent DEvelopment)[32]. To prove the usefulness of context information, we are chosen to compare the performance of our approach against the multicast routing protocols MAODV (Multicast Ad hoc On-demand Vector) [33].

The only reason to choose this protocol from the different routing protocols, that it is the most attractive one based on a study proposed in [34] that compared MAODV with ODMRP [35] and ADMR [36] routing protocols.

Indeed, according to this comparative study, MAODV routing protocol outperforms better than ODMRP and ADMR routing protocols in terms of end-to end delays, throughput and packet delivery ratio.

The simulation parameters are listed in table 2 .

Table 2. Simulation parameters

\begin{tabular}{|l|l|}
\hline Parameters & Value \\
\hline Simulation time & $50 \mathrm{~s}$ \\
\hline Transmission rate & $54 \mathrm{Mbps}$ \\
\hline Playground Dimensions & $1300 \mathrm{~m}$ x 700m \\
\hline Routing protocols & NCABAT, MAODV \\
\hline Number of nodes & 60 \\
\hline Transmission range & $150 \mathrm{~m}$ \\
\hline Mac type & IEEE802.11p \\
\hline
\end{tabular}

\subsection{Performance analysis}

Lots of scenarios were carried out to evaluate our approach. We also intend to study the influence of increasing the network size on the performance of NCABAT as well as MAODV. The performance metrics used for our evaluation were the average of end-to-end delay, throughput and Packet Deliver Ratio (PDR).

\subsubsection{The average of end-to-end delay}

It defines all the delays caused during the routing process such as transmission/re-transmission delays at the MAC layer, transfer delay, route discovery delay.

\subsubsection{Throughput}

Throughput is described as the total number of received packets at destination out of total simulation time. The throughput is the more important metric because we are concerned about the number of transmitted messages. 


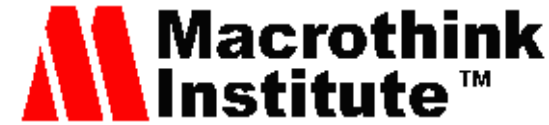

\subsubsection{The Packet deliver ratio (PDR)}

The rate of the number of delivered packets to the best destination defined the Packet deliver ratio (PDR).

\subsection{Results analysis}

\subsubsection{Throughput}

Figure 5 illustrates the results of measured throughput for two previously discussed methods depending on the number of agents. The results depict more efficient behavior of our approach NCABAT in comparison with MAODV when the number of vehicles grows.

As depicted from "Fig. 5", throughput value of MAODV algorithm increases progressively from approximately 200 byte/s at 10 vehicles to 700 byte/s at 50 vehicles and remain stale with increase in the density of vehicles.

However, the throughput of our approach increases from 1400 byte/s to 1800 byte/s. Then, it remains almost constant at [25, 35] vehicles. But, it becomes higher again and reaches 3100 byte/s at 60 vehicles.

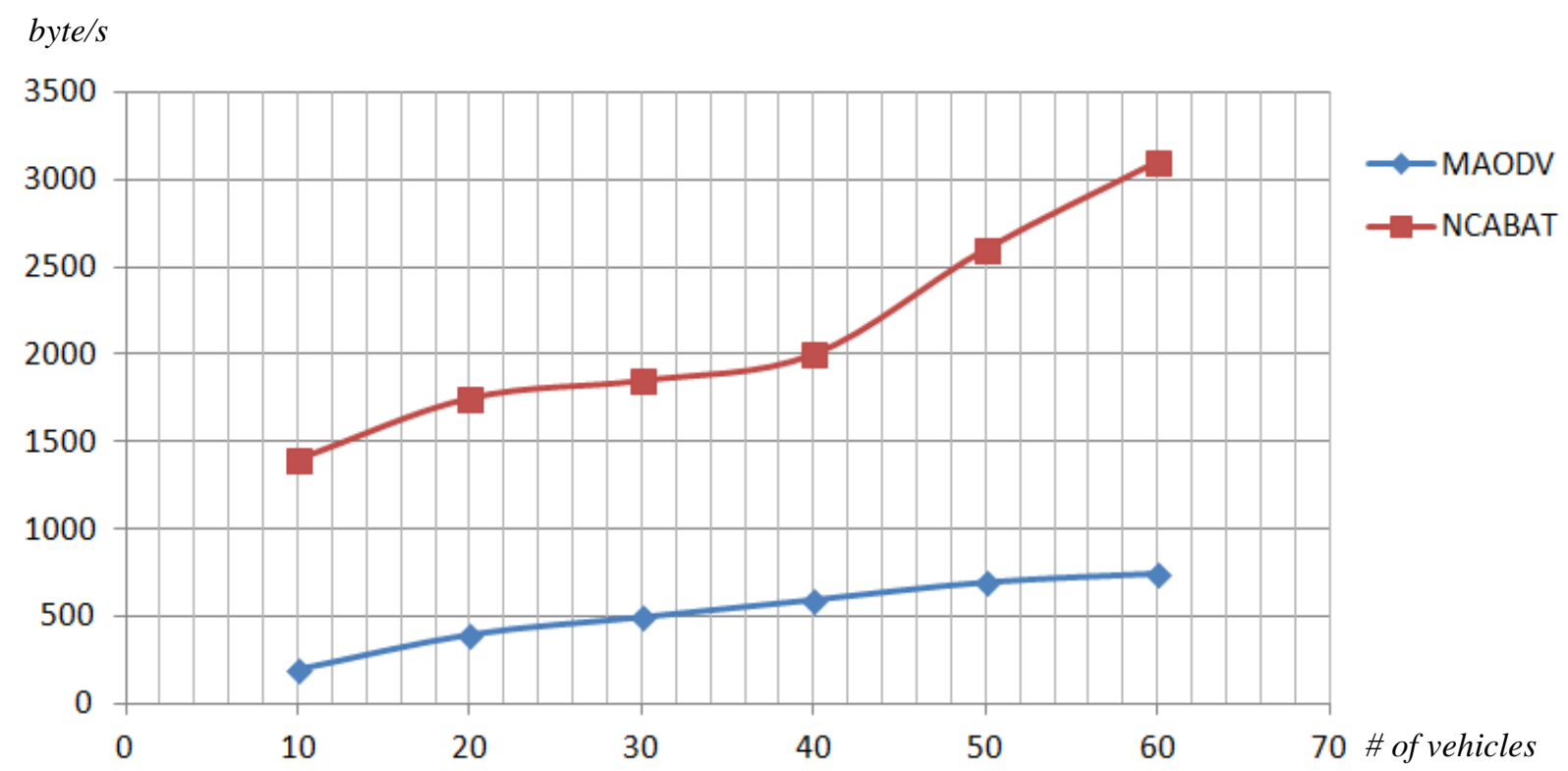

Figure 5. Throughput vs. number of vehicles

\subsubsection{The Packet deliver ratio (PDR)}

"Fig. 6" depicts the average of end-to-end delay measured with increasing the number of vehicles. We can notice that at $[0,20]$ vehicles and although the decrease of end -to-end delay value of NCABAT from 5\% to 3\%, MAODV outperforms our approach NCABAT. Nevertheless, when the number of vehicles grows, our approach demonstrates good scalability. 
In fact, at $[40,60]$ vehicles, our approach decreases to reach $1.7 \%$. However, the average of end-to-end delay of MAODV increases from 3\% at 40 vehicles to $7 \%$ at 60 vehicles.

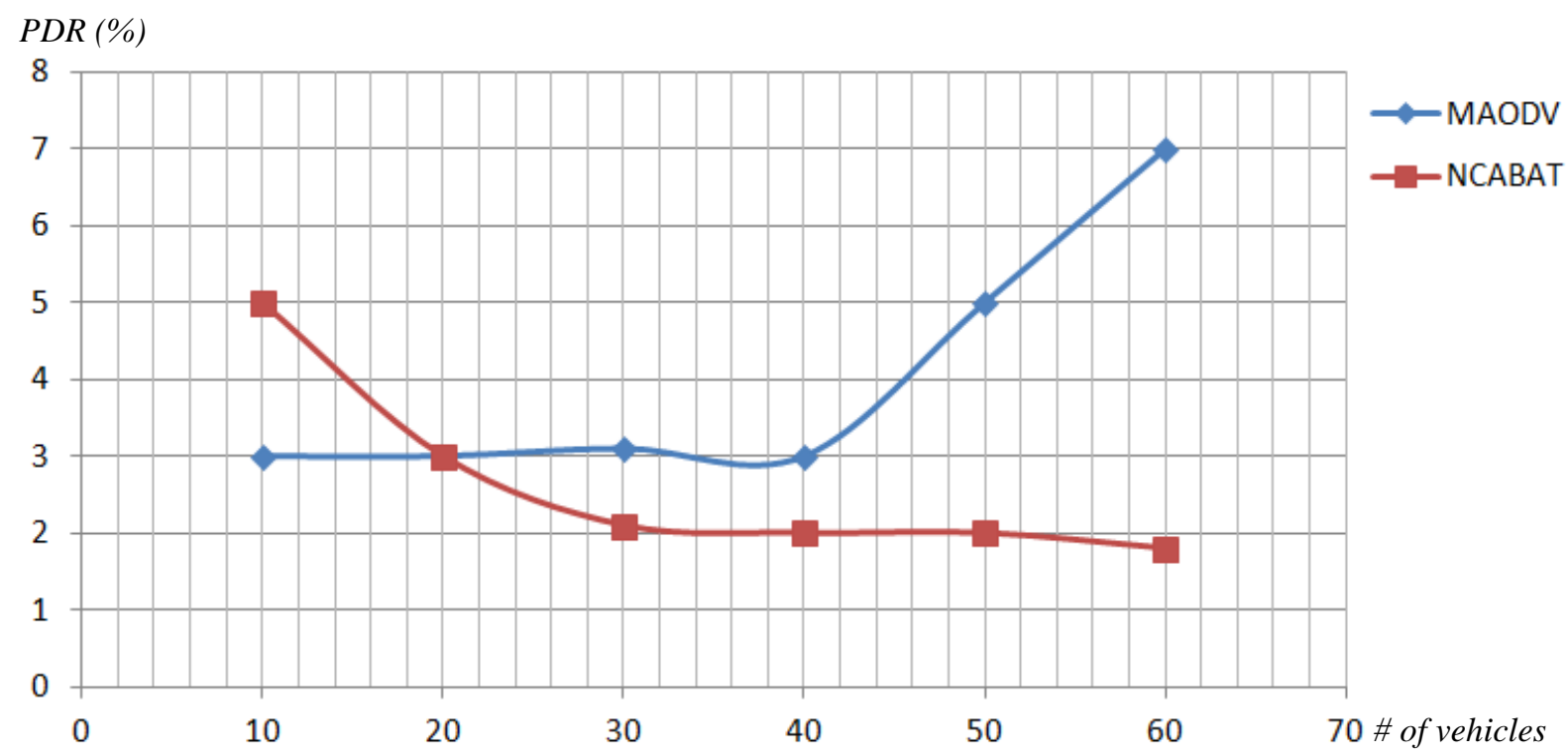

Figure 6. Average of End-To-End delay (EDD) Vs. Number of vehicles

\subsubsection{The Packet deliver ratio (PDR)}

As it can be seen from the "Fig. 7", up to 50 vehicles, the performance of the packet delivery ratio for MAODV is better than our approach. But, when the number of vehicles increases to be 60 vehicles, our approach outperforms MAODV.

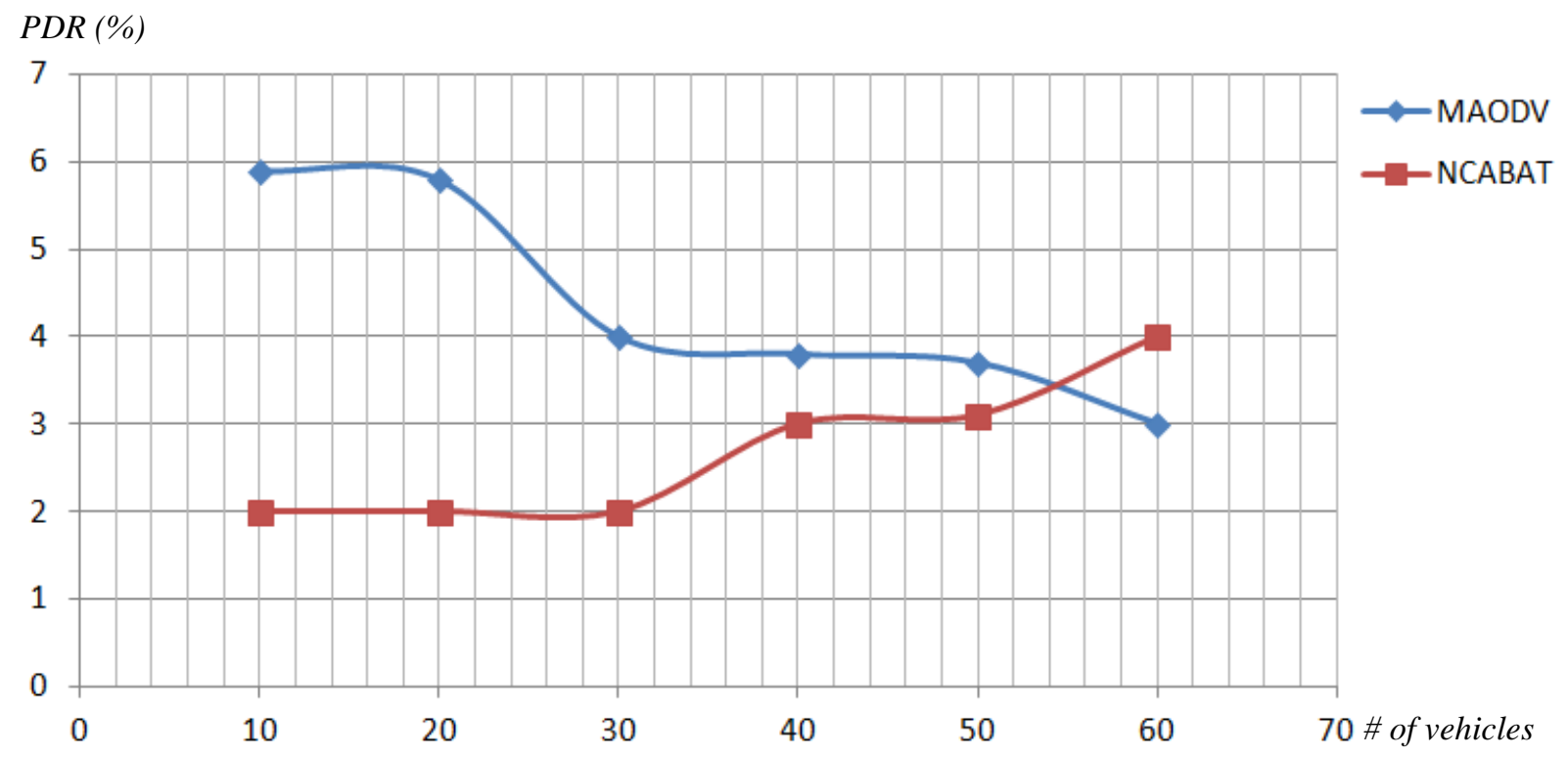

Figure 7. Packet Delivery Ratio (PDR) Vs. Number of vehicles

The table 3 summarizes the performance of our approach against MAODV routing protocol in terms of throughput, average of end to end delay and packet delivery ratio. 
Table3. Numerical comparison between NCABAT and MAODV

\begin{tabular}{|l|c|c|c|c|}
\hline \multirow{2}{*}{} & \multicolumn{2}{|c|}{ Number of vehicles = 10 } & \multicolumn{2}{c|}{ Number of vehicles = 60 } \\
\cline { 2 - 5 } & MAODV & NCABAT & MAODV & NCABAT \\
\hline $\begin{array}{l}\text { Throughput } \\
\text { (byte/s) }\end{array}$ & 200 & 1400 & 700 & 3100 \\
\hline $\begin{array}{l}\text { Average End to } \\
\text { End Delay } \\
\text { (EED) }\end{array}$ & $3 \%$ & $4.5 \%$ & $7 \%$ & $1.7 \%$ \\
\hline $\begin{array}{l}\text { Packet Delivery } \\
\text { Ratio(PDR) }\end{array}$ & $6 \%$ & $2 \%$ & $3 \%$ & $4 \%$ \\
\hline
\end{tabular}

\section{Conclusion}

VANETs possess exceptional network features that distinguish them from MANETs such as highly dynamic topology. From the above mentioned characteristics, it is evident that MANETs clustering algorithms have difficulties in finding stable routing paths in VANETs. Therefore, clustering techniques should be designed to adapt to the VANET environment. These algorithms must take into account all vehicle dynamics.

In this paper, we have proposed a new clustering algorithm based on agent technology. Our main objective is to describe agent properties to vehicles with the purpose of improving traditional schemes in terms of performance. Unlike most previous works, our clustering algorithm tends to group vehicles sharing the same context information (context) during the cluster formation process.

The simulation results show that our approach NCABAT performs well compared to MAODV in terms of throughput, average of end to end delay and packet delivery ratio with increasing in the number of vehicles.

In future work, we intend to study the impact of increasing the velocity value on the performance of our approach.

\section{References}

[1] R. T. Goonewardene, F. H. Ali, and E. Stipidis., "Robust mobility adaptive clustering scheme with Support for geographic routing for vehicular ad hoc networks". IET Intelligent Transportation Systems. Vol. 3, Issue 2, Pp. 148-158, 2009. http://dx.doi.org/10.1049/iet-its:20070052

[2] M. Mottahedi, S. Jabbehdari, S. Adabi, "IBCAV: Intelligent Based Clustering Algorithm in VANET”. International Journal of Computer Science. Vol. 10, Issue 1. Pp538-543. January 2013. 
[3] Samir A. Elsagheer Mohamed, A. Nasr, G. Ahmad Ansari.,” Precise positioning systems for vehicular ad-hoc networks”. International journal of wireless \& mobile networks. Vol. 4, No. 2.Pp251-265. April 2012. http://dx.doi.org/10.5121/ijwmn.2012.4217

[4] N. R. Jennings, K. Sycara, M. Wooldridge, "A roadmap of agent research and development" Autonomous Agents and Multi-Agent Systems. Vol. 1, no 1, 1998, Pp. 7-38.

[5] S. Azzouzi, M. Benattou, M. El Hassan Charaf, J. Abouchabaka, "SMA and Mobile Agents Actors for Distributed Testing”. International Journal of Computer Science. Vol. 7, Issue 5, Pp. 231-238. September 2010.

[6] Mustafa B., Raja U.W., “Issues of Routing in VANET”. Doctoral dissertation, Blekinge Institute of Technology, 2010. Available at: http://denver.bth.se/fou/cuppsats.nsf/all/b1da115d9e5e4a47c1257758 005230c7/\$file/IssuesofroutinginVANETmasterthesis.pdf.

[7] Prabhakar Ranjan, Kamal Kant Ahirwar, "Comparative Study of VANET and MANET Routing Protocols”. Proc. of the International Conference on Advanced Computing and Communication Technologies (ACCT 2011), January 20-22, 2011, pp. 517-523.

[8] A. Rahim, I. Ahmad, Z. S. Khan, M. Sher, M. Shoaib, A. Javed, R. Mahmood, “A comparative study of mobile and vehicular adhoc networks". International Journal of Recent Trends in Engineering. Vol. 2, No. 4. Pp 195-197. November 2009.

[9] Y. Chen, M. Fang, S. Shi, W. Guo, X. Zheng, "Distributed multi-hop clustering algorithm for VANETs based on neighborhood follow”. EURASIP Journal on Wireless Communications and Networking. Vol. 2015, No. 1, Pp 1-12, 2015. http://dx.doi.org/10.1186/s13638-015-0327-0

[10] B. Hassanabadi., C. Shea., L. Zhang., S. Valaee, "Clustering in vehicular ad hoc networks using affinity propagation” Ad Hoc Networks, vol. 13, 2014, pp. 535-548.

[11]M. S. Kakkasageri and S. S. Manvi, "Connectivity and Mobility Aware Dynamic Clustering in VANETs". International Journal of Future Computer and Communication. Vol. 3, No. 1.Pp5-8. February 2014. http://dx.doi.org/10.7763/IJFCC.2014.V3.256

[12]Lloret J., Canovas A., Catala A., Garcia M., "Group-based protocol and mobility model for VANETs to offer internet access". Journal of Network and Computer Applications. Vol. 36, Issue 3, Pp 1027-1038, May 2013.http://dx.doi.org/ 10.1016/j.jnca.2012.02.009

[13]S. Ucar, S. C. Ergen, and O. Ozkasap, "VMaSC: Vehicular Multi-hop algorithm for Stable Clustering in vehicular ad hoc networks". Proc. of the IEEE Wireless Communications and Networking Conference (WCNC 2013), Shanghai, April 7-10, 2013, pp. 2381-2386. http://dx.doi.org/10.1109/WCNC.2013.6554933

[14]H. Idjmayyel, B. R. Qazi, and J. M. H. Elmirghani., "Energy efficient double cluster head routing scheme in a city vehicular network". Proc. of the 27th International Conference on Advanced Information Networking and Applications Workshops (WAINA 2013), Barcelona, 25-28 March 2013, pp. 1594-1599. http://dx.doi.org/10.1109/WAINA.2013.37

[15]S. C. Lo, Y. J. Lin, and J. S. Gao, “A multi-head clustering algorithm in vehicular ad hoc networks,” International Journal of Computer Theory and Engineering, vol. 5, No. 2, pp. 242-247, 2013. 
[16]L. A. Maglaras and D. Katsaros, “Clustering in Urban environments: virtual forces applied to vehicles" Proc. of the IEEE International Conference on Communications Workshops (ICC 2013), Budapest, 9-13 June 2013, pp. 484-488. http://dx.doi.org/10.1109/ICCW.2013.6649282

[17]A. D. Ghodrati, "Reduces Broadcast Storm Using Clustering in VANETs". International Research Journal of Applied and Basic Sciences. Vol, 7, No 13. Pp. 979-987. 2013.

[18]C. Li, C. Zhao, L. Zhu, H. Lin and J. Li, "Geographic routing protocol for vehicular ad hoc networks in city scenarios: a proposal and analysis”. International Journal Of Communication Systems. Vol. 27, Issue 12, Pp 4126-4143, December 2014. http://dx.doi.org/10.1002/dac.2602

[19]Y-S. Chen, Ch-S. Hsu and Ch-H. Cheng, "Network mobility protocol for vehicular ad hoc networks”, International Journal Of Communication Systems. Vol. 27, Issue 11, Pp: 3042-3063, November 2014. http://dx.doi.org/10.1002/dac.2525

[20]W. Xiaonan and Q. Huanyan, "Constructing a VANET based on cluster chains”.International Journal Of Communication Systems, Pp 2497-2517, Vol. 27, Issue 11, November 2014. http://dx.doi.org/10.1002/dac.2484.

[21]Ch-Ho. Ou, “A roadside unit-based localization scheme for vehicular ad hoc networks”. International Journal Of Communication Systems. Vol. 27, Issue 1, Pp 135-150, January 2014. http://dx.doi.org/10.1002/dac.2352.

[22]A. Daeinabi, A. G. P. Rahbar, A. Khademzadeh., "VWCA: An efficient clustering algorithm in vehicular ad hoc networks". Journal of Network and Computer Applications. Vol. 34, Issue 1, Pp. 207-222, January 2011.

[23]Q. Yang, A. Lim, S. Li, J. Fang, and P. Agrawal. "ACAR: Adaptive Connectivity Aware Routing for Vehicular Ad Hoc Networks in City Scenario", Mobile Networks and Applications (MONET). vol. 15, no. 1, pp. 36-60, 2010. http://dx.doi.org/10.1007/s11036-009-0169-2

[24]J. Lloret, M. Garcia, D. Bri, J.R. Diaz, A cluster-based architecture to structure the topology of parallel wireless sensor networks, Sensors 9 (12). Pp. 10513-10544. 2009.

[25]S. Harrabi, W.Chainbi, K.Ghédira, "A multi-agent proactive routing protocol for Vehicular Ad-Hoc Networks". Proc. of The 2014 International Symposium on Networks, Computers and Communications (ISNCC 2014 ), Hammamet-Tunisia, 17-19 June 2014. http://dx.doi.org/10.1109/SNCC.2014.6866523.

[26]S. Harrabi, W. Chainbi, K. Ghédira, "A Multi-agent Approach for Routing on Vehicular Ad-Hoc Networks", Procedia Computer Science. Vol. 19, Pp 578-585, 2013. http://dx.doi.org/10.1016/j.procs.2013.06.077

[27]G. Chen, D. Kotz, “A survey of context-aware mobile computing research". Vol. 1. No. 2.1. Technical Report TR2000-381, Dept. of Computer Science, Dartmouth College, 2000.

[28]N. Kumar, N. Chilamkurti, J. H. Park, "ALCA: agent learning-based clustering algorithm in vehicular ad hoc networks", Personal and Ubiquitous Computing, Volume 17, Issue 8, pp 1683-1692, December 2013. http://dx.doi.org/10.1007/s00779-012-0600-8 
[29]S. Saharan and R. Kumar, "QoS Provisioning in VANETs using Mobile Agent”, International Journal of Computer Science \& Communication. Vol. 1, Issue. 1. Pp. 199-202. January-June 2010.

[30]Ramesh B. Koti and Mahabaleshwar S. K., "Multi Agent Based Congestion Control in VANETs". International Journal of Future Computer and Communication. Vol. 3, Issue 2. Pp. 102-104. April 2014.

[31]Gilbert G. Chen, Joel W. Branch, and Boleslaw K. Szymanski, “A Self-selection Technique for Flooding and Routing in Wireless Ad-hoc Networks”. Journal of Network and Systems Management. Vol. 14, Issue 3. Pp. 359-380. September 2006.

[32]Fabio Bellifemine, Agostino Poggi, Giovanni Rimassa, "Developing multi-agent systems with a FIPA-compliant agent framework”. Software - Practice and experience. Vol. 31, Issue 2, Pp. 103-128, 2001. http://dx.doi.org/10.1002/1097-024X(200102)31:2<103::AID-SPE358>3.0.CO;2-O.

[33] E. M. Royer, C. E. Perkins, "Multicast Ad hoc On-Demand Distance Vector (MAODV) Routing”. Internet Draft: draft- ietf-manet-maodv-00.txt, 2000.

[34]Jorjeta G. Jetcheva, David B. Johnson., "A Performance Comparison of On-Demand Multicast Routing Protocols for Ad Hoc Networks", Technical Report CMU-CS-04-176, School of Computer Science, Carnegie Mellon University, December 2004.

[35] Sung-Ju LEE, William SU, Mario GERLA, On-demand multicast routing protocol in multihop wireless mobile networks. Mobile networks and applications, vol. 7, no 6, pp. 441-453, 2002. http://dx.doi.org/10.1023/A:1020756600187

[36]J. G. Jetcheva and D. B. Johnson, “Adaptive demand-driven multicast routing in multi-hop wireless ad hoc networks", Proc. of the 2nd ACM international symposium on Mobile ad hoc networking \& computing (MobiHoc 2001), New York, NY, USA, 2001. http://dx.doi.org/10.1145/501422.501423

\section{Copyright Disclaimer}

Copyright reserved by the author(s).

This article is an open-access article distributed under the terms and conditions of the Creative Commons Attribution license (http://creativecommons.org/licenses/by/3.0/). 